\title{
Las "Redes educativas regionales; un marco integral de gestión del conocimiento, para la reforma universitaria que contribuye a la transformación nacional"
}

Céleo Emilio Arias ${ }^{1}$

\begin{abstract}
"Cuando logramos conectarnos con la grandeza y maravilla de nuestros sueños para el bien común, entonces sentimos que estamos contribuyendo a las redes generacionales". Cejas'92
\end{abstract}

\section{RESUMEN}

La gestión del conocimiento a través de redes educativas regionales, es un mandato y política institucional que se construye mediante procesos participativos incluyentes durante el proceso actual de reforma universitaria. Este mecanismo permite la "organización de la nueva estructura universitaria" creando oportunidades para deconstruir la actual estructura centralizada, burocratizada, altamente ineficiente y poco transparente de la UNAH, generando nuevas formas de organización para gestionar nuevas respuestas educativas a las demandas sociales autenticas de una nueva institucionalidad pública, en donde la vinculación de la Universidad con la sociedad sea de mutuo aprendizaje y enriquecimiento, para que la UNAH aprenda sobre los nuevos movimientos sociales. Las unidades académicas están iniciando su participación amplia y creativa para impactar positivamente en el desarrollo, abriéndose a esquemas de gestión compartida, complementaria y sinérgica.

Las redes tienen un sentido: contribuir a resolver los problemas nacionales y regionales; tienen un contenido que se definirá desde el desarrollo curricular de los campos del conocimiento que demandan las necesidades y potencialidades del Desarrollo Humano Sostenible (DHS), y tienen una forma (región) definida por las capacidades y potencialidades de la estructura institucional, y por la articulación con los otros niveles del sistema educativo nacional.

Las Redes Regionales son organizaciones de coordinación de las diferentes unidades académico-administrativas a lo interno de la UNAH en las diferentes regiones del país para cumplir con los criterios de calidad, pertinencia y equidad,

\footnotetext{
${ }^{1}$ Vice-Rectoría Académica-UNAH, celeoarias@yahoo.com
} 
permitiendo un marco integral para el quehacer universitario en investigación, docencia y vinculación con la sociedad, para dar respuesta a las necesidades autenticas de la nación y contribuir a desplegar las potencialidades del Desarrollo Humano Sostenible, contribuyendo de esta manera a la viabilidad de visión de país (2010-2038) y plan de nación (2010-2022).

Palabras Clave: Procesos participativos incluyentes; demandas sociales autenticas; nueva institucionalidad publica; gestión compartida complementaria y sinérgica; desarrollo curricular; calidad, pertinencia y equidad; visión de país; plan de nación.

\section{ABSTRACT}

Knowledge management through regional educational networks, is a mandate and institutional policy that builds through inclusive participatory processes during the ongoing process of university reform, a mechanism that allows the "organization of the new university structure" creating opportunities to deconstruct the current centralized, bureaucratic, inefficient and no transparency of the UNAH; generating new forms of organization to manage new educational responses to social demands authenticity of a new public institutions, where the connections between the University and society is mutual learning and enrichment for the UNAH learn about the new social movements, the academic units are starting their broader creative participation to positively impact the development, opening a shared management schemes, complementary and synergistic.

The networks have a meaning: to help solve national and regional problems, are content to be defined from the curriculum development of fields of knowledge demanded by the needs and potential of the DHS, and have a shape (region) defined by the capabilities and potential of the institutional structure, and articulation with other levels of the national education system.

Regional networks are organizations coordinate the various academic and administrative units to internal UNAH in different regions of the country to meet the criteria of quality, relevance and equity. Allowing a comprehensive framework for university activities in research, teaching and relationship with society, to meet the genuine needs of the nation and contribute to the potential deployment of sustainable human development, thus making possible linkage with the national vision (2010-2038) and national plan (2010-2022). 
Keywords: Inclusive participatory processes; social demands authentic; new institutional publishes; shared management complementary and synergistic; curriculum development; quality, relevance and equity; vision of country; nation plan. 


\section{INTRODUCCIÓN Y ANTECEDENTES DE LAINICIATIVA}

La "organización de la nueva estructura universitaria" crea oportunidades para deconstruir la actual estructura centralizada, burocratizada, altamente ineficiente y poco transparente de la UNAH; es el momento de generar nuevas formas de organización para gestionar nuevas respuestas educativas de la UNAH a las demandas sociales de una nueva institucionalidad pública, en donde la vinculación de la Universidad con la sociedad sea de mutuo aprendizaje y enriquecimiento, para que la UNAH aprenda sobre los nuevos movimientos sociales y asuma la diversidad organizativa como inherente a la dinámica social y de las instituciones. Las Facultades y los Centros Universitarios Regionales deben participar amplia y creativamente en esta corriente renovadora para impactar positivamente en el Desarrollo Humano Sostenible (DHS), abriéndose a esquemas de gestión compartida, complementaria y sinérgica cuando la realidad así lo reclame.

El proceso que se detalla en el presente artículo, permite identificar el proceso de cadena de valor de una política institucional de gestión del conocimiento, derivada de la visión, misión estratégica y de un enfoque de mando integral estratégico.

Se describe de forma breve algunos factores determinantes del proceso; se explican los antecedentes de la iniciativa, luego algunos conceptos básicos, así como la justificación de dicho emprendimiento en términos de cobertura y acceso a la educación superior; también se describen aspectos relevantes del análisis de situación de unidades académicas regionales.

Se enuncia el fin, propósito y objetivos, así como las políticas regionales, su organización y función de los órganos de coordinación; la gestión del desarrollo de las redes para lograr su implementación, dándole visibilidad a los avances que se están logrando; los principales retos que se necesitan lograr, así como las conclusiones al estado actual para lograr un impulso más significativo de la iniciativa.

\section{CONCEPTOS BASICOS DE LAS REDES EDUCATIVAS REGIONALES DE LA UNAH.}

La "gestión del conocimiento" como proceso humano y educativo, según Sveiby (2001) uno de los fundadores de la idea sobre la Gestión del Conocimiento, anota que ésta se encuentra constituida por dos "rutas": la ruta de la tecnología de la información (TI) la cual es Gestión de la Información, y la ruta del recurso humano, la cual es la gestión humana. Pero también aborda y examina los problemas de la 
inserción social del conocimiento, los nuevos procesos en la generación y transformación del conocimiento en prácticas profesionales y sociales y la transferencia de tecnología social a las universidades, instituciones y sociedad.

El conocimiento tácito, tal como fue inicialmente definido por Polanyi, es el conocimiento personal escondido, aún de la consciencia del conocedor, que no puede ser "capturado" sino únicamente demostrado a través de nuestro conocimiento que puede expresarse a través de nuestros actos. Nonaka y Takeuchi expandieron este concepto incluyendo el conocimiento previamente no expresado, el conocimiento expresable (información), el cual fue llamado por Wilson como conocimiento implícito, o la parte del conocimiento tácito que puede ser compartido. El conocimiento explícito es aquel componente codificado y trasmitido en un lenguaje sistemático y formal, como por ejemplo: los documentos, las bases de datos, el Internet, el correo electrónico, etc.

Se enfatizó en las discusiones la necesidad de considerar el término conocimiento autóctono, el cual también es llamado conocimiento local o tradicional. El creciente interés por este conocimiento está muy relacionado con el giro que se ha dado en el propio desarrollo de la ciencia que reconoce que "el conocimiento autóctono es el conocimiento local que es único o propio de una cultura o sociedad en particular. Es la base para la toma de decisiones a nivel local".

\section{CARACTERÍSTICAS Y PRINCIPALES RASGOS DE LAS REDES}

- Relacioneshorizontales

- Relaciones de telaraña inter organizacionales

- Interacción con base en relaciones relativamente sueltas

- Interdependencia recíproca

- Contribución eficiente y aceptada

- Intercambiar continuamente experiencias

- Desarrollar estructuras de consenso y compromiso

\section{JUSTIFICACIONES PARA LA GESTIÓN DEL CONOCIMIENTO A TRAVÉS DE REDES EDUCATIVAS REGIONALES}

\section{Situación general de la cobertura y acceso a la unah.}

Si consideramos la situación actual de la educación superior en Honduras, y basándonos en un análisis de la cobertura de la UNAH a nivel nacional y de la 
equidad en el acceso de la población, se puede identificar que la UNAH tiene una cobertura limitada al llamado "eje central del desarrollo", sin presencia en las regiones más pobres del país; se confirma con los datos de acceso que reflejan entre otros, que en el año 2007 no ingresó a la UNAH ni un solo estudiante de los 46 municipios más postergados, situación que se mantiene a lo largo de la última década; se podría traducir como un indicador de la falta de equidad en el acceso a la oferta educativa de la UNAH. A lo anterior se suma que el Nivel de Educación Superior en el país no ha logrado aumentar su cobertura en función de atender la creciente demanda de la población, sobre todo de los/las jóvenes, por los servicios de educación superior, manteniéndose el índice de cobertura en 14.3\% del 2002 hastala fecha.

\section{Aspectos relevantes del análisis de situación de los centros universitarios regionales (cur) y centros asociados de educación a distancia (casued).}

Del análisis situacional de los CUR's, encontramos profundas desigualdades y pobreza creciente; en el caso de los CASUED, se identificó entre otros: (1) Limitada 0 nula experiencia para formación en Educación a Distancia (EDA), y las Tecnologías de Información y Comunicación (TIC); (2) Ausencia de un marco de políticas institucionales en EDA; brinda una oferta académica muy baja; (3) Históricamente se ha mantenido con el mismo funcionamiento con ausencia de innovaciones educativas sin propuestas académicas fundamentadas; (4) El currículo es cerrado e inflexible en las carreras que se ofertan; (5) Los planes de estudio no han sido revisados ni actualizados de acuerdo a los requerimientos y necesidades de la educación superior en las carreras ofrecidas; (6) Deficiencia en infraestructura física y tecnológica; (7) Poca integración entre las tareas académicas de docencia, investigación y vinculación. (ver Mapa1)

\section{Descripción de la iniciativa}

Las situaciones anteriores demandan de la UNAH, una propuesta que busque como fin contribuir desde la UNAH al Desarrollo Humano Sostenible (DHS) regional, y a la superación de los problemas prioritarios regionales y nacionales con un enfoque de gestión estratégica (integrar acciones y recursos de forma sinérgica y complementaria) y la organización de redes regionales; como propósito, contribuir a mejorar la pertinencia de la educación universitaria y la equidad en el acceso de la población hondureña a la educación superior. Dentro de los objetivos se persigue: (a) Lograr mayor calidad (eficiencia, eficacia y efectividad) y transparencia en el uso de los recursos institucionales, en función de atender las necesidades y demandas prioritarias de la población en el campo de la educación 
superior, a nivel regional; (b) Reorganizar los CUR's y los CASUED's a partir de un modelo de gestión más pertinente del conocimiento en respuesta a los problemas regionales; (c) Generar respuestas coherentes con la naturaleza de la UNAH, a las demandas por mayor y mejor educación superior que los actores regionales le presentan a la Universidad; (d) Operacionalizar el modelo educativo de la UNAH a través de programas universitarios de formación, investigación, y vinculación universidad-sociedad, que contribuyan a la solución de los problemas prioritarios regionales;(e) Promover la cooperación solidaria y la complementariedad de la oferta educativa de la UNAH, a nivel regional y nacional; (f) Fortalecer la capacidad de gestión académica y administrativa financiera de las unidades académicas regionales.

\section{BENEFICIOS DE LAS REDES}

En el proceso de análisis de construcción participativa con los/las Directores/as de los CUR's se han identificado los siguientes beneficios o bondades de trabajar la gestión del conocimiento en redes, basado en el análisis de otras redes sectoriales y el involucramiento directo de la UNAH: (a) Permiten la creación de espacios interdisciplinarios; (b) Facilitan el papel de la UNAH como agente de cambio y de desarrollo humano sostenible; (c) Generan mayor certeza en las acciones; (d) Mejor aprovechamiento de los recursos disponibles; (e) Aprendizaje permanente e intercambios; (f) Ganamos conectividad y competitividad como institución; (g) Mayor cobertura de la acción universitaria; (h) Mayor equidad en la oferta educativa a nivel nacional.

\section{Políticas de redes educativas regionales}

Entre algunas políticas se identifican las siguientes: (a)Toda red estará integrada por un CUR como nodo central, uno o más Institutos Tecnológicos, y uno o más Centros de Recursos de Aprendizaje para la Educación a Distancia, Institutos de Investigación, y Centros Experimentales orientados a la necesidades y demandas estratégicas del desarrollo regional e interregional; (b) Dichos nodos deben contribuir a resolver los problemas nacionales y regionales y al logro de la calidad, la pertinencia y la equidad; (c) Definir una estructura organizacional que permita el desarrollo de los nodos de la red, tanto a lo interno de la institución, como a lo externo; (d) Definir el contenido de la redes, desde el desarrollo curricular de los campos del conocimiento que demandan las necesidades y potencialidades del Desarrollo Humano Sostenible; (e) Fomentar el proceso de construcción del conocimiento considerando cuatro aspectos: gestión de la información, intercambio de conocimientos, aspectos organizacionales y aspectos culturales; (f) Definir las 
redes por las capacidades y potencialidades de la estructura institucional, por la articulación con los otros niveles del sistema educativo nacional y, por los problemas, necesidades y potencialidades comunes a espacios población; (g) Orientar el desarrollo de las redes desde una perspectiva estratégica, considerando que debe: (1) Dar respuesta a problemas actuales y potenciales del desarrollo regional, con una visión sistémica de país, de Centro América y del mundo; (2) Revalorizar la historia. (3) Ser incluyente del conocimiento universal, nacional, regional y local y, crear espacios y medios de comunicación entre el saber científico y el saber popular; (4) Articular adecuadamente lo general, lo particular y lo específico de las disciplinas y las ciencias en un ejercicio permanente de multidisciplinariedad e interdisciplinariedad; (5) Contribuir a reducir las brechas en el acceso a la información y a las tecnologías; (6) Impulsar un sistema de incentivos; (7) Establecer un sistema articulado de monitoria y evaluación.

\section{ORGANIZACIÓN DE LAS REDES EDUCATIVAS REGIONALES}

Las Redes Regionales son organizaciones de coordinación de las diferentes unidades académico-administrativas que la UNAH tiene a lo interno de la institución en las diferentes regiones del país para cumplir con los criterios de calidad, pertinencia y equidad de su oferta académica. La organización propuesta de las Redes Regionales de la UNAH es la siguiente: (1) Órgano de Coordinación (Consejo Directivo Regional; (2) Órgano Ejecutivo (Dirección del Centro Universitario Regional); (3) Órgano de Asesoría (Comité Regional Asesor); (4) Órganos de Coordinación Operativa (Comité de Coordinación Docente, Comité de Coordinación de Investigación Científica, Comité de Coordinación Vinculación, Comité de Coordinación de Gestión Académica; (5) Órganos Operativos de Base (Centro Universitario Regional, Institutos Tecnológicos, Institutos de Investigación, Centro de Recursos de Aprendizaje a Distancia, Centros Experimentales).

\section{GESTIÓN DEL DESARROLLO DE LAS REDES}

Algunos de los mecanismos propuestos por los/las Directores/as de los CUR's para el desarrollo de las redes regionales son: Complementariedad de recursos, desarrollo curricular articulado, formación de docentes con las nuevas competencias exigidas para la gestión del conocimiento en red, aproximaciones con actores claves en todos los niveles de la UNAH y fuera de la UNAH, acompañamiento y monitoria, mejorar la gestión administrativa y financiera, cambio de actitud con sentido académico, sistematización y divulgación permanente del conocimiento generado en las redes, promover cambios en los instrumentos regulatorios (académicos y laborales). 
El ciclo sistemático de la gestion del conocimiento (ver gráfico 1) se aplicó en una primera aproximación en el sub-sector de Agua y Saneamiento, lo cual permite tener un referente que está contextualizado en una arquitectura multisectorial. El siguiente gráfico muestra los diferentes elementos que se consideran en el ¿cómo? de la gestión del conocimiento en redes regionales.

\section{¿Como se va a gestionar el conocimiento en las Redes Regionales?}

El diagrama siguiente esquematiza el proceso de construcción de conocimiento que se podría considerar para realizar análisis temáticos a partir de la problematización de la realidad regional.

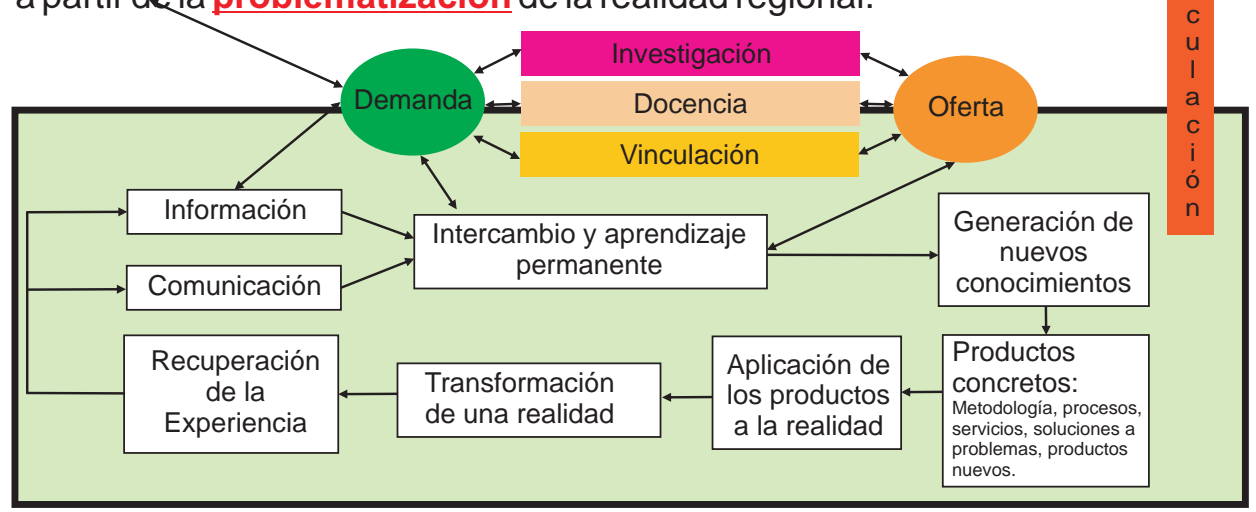

Gráfico 1: Fuente de elaboración

\section{MOMENTOS EN LA GESTIÓN DEL CONOCIMIENTO}

I. Generación: se deben responder las siguientes interrogantes;¿Qué conocimiento?, ¿para qué? ¿cómo?, ¿qué fuentes?

II. Organización y administración: a través de currículos y planes de estudio, prácticas docentes y aprendizaje formal e informal.

III. Aplicación o utilización: Formas y medios de divulgación, acceso y solución 0 superación de problemas.

Los modelos de excelencia en la gestión del conocimiento son un campo de acción para los científicos de todas las áreas del conocimiento; el quehacer universitario requiere de fortalecer líneas de investigación y vinculación con la sociedad, así 
como también con la nueva oferta integral de carreras universitarias a los diferentes grados: Grado-Asociado, Licenciaturas, Especialidades, Maestrías, Doctorados y Post-Doctorados.

La iniciativa de las Redes Educativas Regionales se enmarca en sistemas de gestión de aseguramiento de la calidad con estándares internacionales; es consistente con el enfoque de plan de nación, no obstante el siguiente paso sería articular el sistema de gestión del conocimiento de la visión de país (2010-2038) y plan de nación (2010-2022) con el rol que le corresponde por mandato legal a la UNAH, como institución líder del nivel de educación superior; le compete la identificación participativa con los actores claves de los temas y áreas prioritarias autenticas de los problemas y potencialidades de cada una de las regiones, así como también la generación y luego distribución y divulgación de la gestion del conocimiento en toda su cadena de valor. El hexágono de la competitividad sistémica identifica con buen nivel de detalle el rol que le corresponde a las universidades.

La viabilidad de lograr una gestión integral (planificación, implementación, monitoria y evaluación) sostenible de la política, dependerá de varios supuestos y mecanismos de soporte, apoyo, cooperación con un enfoque ganar/ganar. Que los actores claves como: Sindicato, Asociación de Estudiantes y Docentes, Asociación de padres de familia, Junta de Dirección Universitaria (JDU), Consejo Universitario, Gabinete Rectoral, Sociedad Civil, entre otros actores claves, se logren articular alrededor de agendas compartidas con objetivos comunes; pero además que se logre establecer y consolidar un sistema de monitoria y evaluación articulado de forma horizontal y vertical.

\section{Avances de las redes educativas regionales}

Los avances en el contexto nacional son diversos y heterogéneos; algunas regiones están avanzando más que otras; entre las acciones que están logrando mayor madurez, por ejemplo: el tema de identificar la demanda autentica integral de servicios universitarios en los quehaceres de docencia (nuevas carreras profesionalizantes), investigación (identificar las líneas prioritarias de cada región) y vinculación con la sociedad (líneas prioritarias de cooperación). En la matriz 1 se muestran los avances de cada etapa y fases del plan de implementación y desarrollo de las redes.

De los pasos a seguir para implementar la propuesta, entre otros: (1) Socializar la 
propuesta con todos los actores claves; (2)Aprobación del programa presupuestario de creación del sistema de redes por parte de la JDU-UNAH; (3) Diseñar una estrategia integrada de movilización de recursos (financieros, humanos, tecnológicos, entre otros) entre la UNAH, la sociedad civil organizada, sector público y privado y la cooperación internacional, orientada a lograr sinergias y complementariedad en el desarrollo de las capacidades de gestión del conocimiento de los nodos de la red.

Algunos temas identificados se logran enmarcar en los objetivos de desarrollo del milenio (ODM) y los pilares del hexágono de la competitividad sistémica: Innovación, Investigación y Desarrollo (I+D+I), articulación con el programa nacional de competitividad en la fase 2007-2009 y articulación y alineamiento estratégico interinstitucional para fortalecer los pilares de Innovación y el pilar de educación superior; fase 2010-2014.

Otro de los temas claves es el manejo costero integrado en el marco de grandes ecosistemas marinos (Large Marine Ecosystem), en alianza con iniciativas Latinoamericanas, lideradas por la Universidad de Rhode Island y el Centro de Recursos Costeros de Guayaquil, y la Organización de las Naciones Unidas para el Desarrollo Industrial (ONUDI).

La matriz 2 muestra los resultados obtenidos del primer ciclo de gestión del conocimiento aplicando todos los elementos teóricos (enfoque integral); se logró implementarlos, pasando por toda la cadena de valor del conocimiento del subsector de agua y saneamiento básico. La UNAH participó como un actor clave de la Red, permitiendo generar buenas prácticas e implementar acciones significativas en uno de los campus regionales (CURLA); el Centro de Recursos del Conocimiento del sub-sector de agua y saneamiento, continuará con el apoyo de otras áreas y temáticas prioritarias de la red del Atlántico y además apoyando la apertura de otros centros de recurso en diferentes regiones en el marco de la redes educativas.

Otro de los avances determinantes es el diseño del programa de formación permanente de gestores del conocimiento en redes regionales, el que se conceptualizará y sistematizará en módulos claves, considerando los siguientes elementos: Liderazgo (modelo teórico transformacional, transaccional con créditos a la idiosincrasia local, autóctona tácita y explicita), gestión por resultados, diagnósticos regionales, nuevo modelo educativo (diseños curriculares), gestión, planificación, monitoria y evaluación universitaria articulada, gerencia de redes para la gestión del conocimiento, aseguramiento de la calidad-mejora continua, 
sistematización de experiencias, administración financiera integral por resultados, unidades gestoras de programas y proyectos. Otro diseño avanzado que contribuye y complementa positivamente, es el del sistema articulado de planificación, monitoria y evaluación, que según la experiencia de la reforma mexicana universitaria, es un factor determinante; además esta herramienta permite pasar del discurso a las acciones para obtener productos, resultados e impactos, en el corto, mediano y largo plazo.

En base a una ruta crítica se ha logrado avanzar en el año 2009 en el diseño del estudio de oferta integral de servicios universitarios de la UNAH; esta iniciativa la lidera el Instituto de Investigaciones Económicas y Sociales y servirá como insumo para la priorización de las áreas autenticas del conocimiento demandadas por la sociedad. Se está hibridando una metodología rigurosa cuantitativa y cualitativa e integrando un enfoque de investigación acción al desarrollo, que permita ir generando e integrando al proceso los aspectos y factores analizados más determinantes.

Se han identificado en el proceso algunos temas claves, como es el caso del subsector agua y saneamiento básico, que ya logró implementar un ciclo de gestión del conocimiento durante un proceso de 9 año; se logra identificar la aplicación teórica a una demanda autentica de la sociedad, generando conocimiento y respuestas traducidas en carreras a nivel de tecnólogo, diseños curriculares en proceso de viabilizarse a nivel de Maestría y Doctorado, investigación aplicada a una serie de subtemas; gobernabilidad, tecnologías apropiadas en uso y manejo de aguas residuales; se ha logrado una generación de documentos de la sistematización de esta experiencia la cual logró transversalizar la temática con los objetivos, metas e indicadores del Desarrollo del Milenio(ODM),(ver Matriz 2)

Otros temas que se han venido trabajando en el Postgrado Latinoamericano de Trabajo Social y Gestión del Desarrollo, se articulan con las siguientes líneas de investigación de centros internacionales:

\section{- Creación sostenible de valor para trabajo decente-digno}

Este grupo se centra en las estrategias para aumentar la creación de valor mediante el desarrollo de tecnologías para un uso más eficiente y sostenible de los recursos y la mejora de las capacidades humanas, preferentemente a lo largo de las cadenas de productos agroindustriales o servicio. Los gestores académicos deben estar interesados en el análisis espacial y sectorial detallando los vínculos urbanorurales, para atender la movilidad de personas, bienes, servicios, transferencias de 
recursos, la información y los flujos asociados de nutrientes, energía y agua. Hay algunas iniciativas en marcha como por ejemplo; manufactura de alto rendimiento (HPM, por sus siglas en Inglés) aplicado a MIPYMES, observatorio económico (estos temas son liderados por el Instituto de Investigaciones Económicas y Sociales como se mencionó anteriormente), análisis de cadenas de valor en varios subsectores de la Red del Atlántico (Biocombustibles en base a Palma Aceitera, Piñón, Etanol de caña de azúcar, las valoraciones socio-económicas para contribuir al sistema de pagos por servicios ambientales es otro de los sub-temas a continuar fortaleciendo por parte de la UNAH.

Ya existen experiencias piloto en Honduras de mecanismo limpio de bonos de carbono con instituciones suizas, además definición de tarifas de uso y manejo del agua; varios Centros Universitarios Regionales han desarrollado experiencias y están en el proceso de intercambios con otras unidades académicas. Formamos parte de la Red Mesoamericana de Investigación y Desarrollo de Biocombustibles, en alianza con Zamorano, FHIA, Empresa Privada, EMBRAPA; se están gestando una diversidad de acciones prioritarias.

- Instrumentos para la promoción de trabajo decente-digno

Esta línea se centra en la eficacia de los instrumentos existentes en la aplicación de las normas mínimas de trabajo formulado por la Organización Internacional del Trabajo (OIT), tales como la contratación pública, gestión de la cadena de suministro, y los salarios mínimos nacionales.

Los gestores académicos deben estar interesados en el análisis de esos instrumentos y su aplicación efectiva en contextos de estado o región diferente. Se prestará especial atención en dirigirse a su impacto o importancia para las relaciones de trabajo informales y en particular sobre las condiciones de trabajo y la vida de los trabajadores migrantes temporales. De los ejemplos en esta línea se han realizado estudios de las condiciones de trabajo en la industria de la maquila, trabajos de tesis realizados en el marco de la Maestría Latinoamericana de Trabajo Social (MLATS) con orientación en gestion del desarrollo.

- Estrategias de empoderamiento para trabajo decente

Esta línea se centra en la promoción de las buenas condiciones de trabajo en decisiones económicas y políticas, los procesos de adopción en el contexto de erosión de las capacidades de los trabajadores a participar en la acción colectiva debido a la naturaleza informal cada vez mayor de trabajo. Con ello se presta 
especial atención a los grupos especialmente vulnerables, como los trabajadores domésticos. Los gestores académicos deben estar interesados en explorar las posibles fuentes y formas de organización para promover estrategias de capacitación en relaciones laborales informales.

Es importante mencionar que existen una serie de temas que se vienen trabajando en varias unidades académicas; se está trabajando en un inventario de iniciativas como primer paso, para luego articularlas alrededor de los temas prioritarios, los cuales se enmarcan en varios niveles de autenticidad y prioridad en ámbitos internacionales, nacionales, regionales y locales.

El siguiente paso será el alineamiento con el enfoque de Redes Educativas Regionales para la gestion del conocimiento, además en el Plan de Nación de Honduras y los Objetivos de Desarrollo del Milenio. Los momentos de socialización de la iniciativa permitirán ir apropiando a la sociedad y realizar auditorías sociales para identificar oportunidades de mejora continua y rendición de cuentas.

El sub-sistema de monitoria y evaluación de la Vice RectoríaAcadémica ha logrado avances significativos; actualmente se cuenta con el diseño de sistema integral de planificación, seguimiento, monitoria y evaluación en los centros universitarios regionales en el marco de las redes educativas de la UNAH; este sistema permitirá la gestion del conocimiento de las Redes.

Los productos, resultados e impactos de la gestion de las Redes, se dará en diferentes niveles de umbral de desempeño (Umbral Pobre Desempeño, Umbral Aceptable Desempeño, Umbral Buen Desempeño y Umbral Excelente Desempeño) dependiendo de la dinámica que le impregnen los diferentes actores, desde la asignación presupuestaria hasta la actitud de los gestores administrativos y académicos, es decir que pasa por todos los factores y componentes (eslabones) de la cadena de valor académica con enfoque integral de gestión del conocimiento.

\section{Retos}

Lograr la madurez institucional a lo interno en las diferentes unidades académicas, orientada a diseñar agendas comunes y compartidas y sobre toda articulación complementaria y sinérgica (ver grafico 2). Sistemas automatizados de gestión por resultados que permitan orientar la administración financiera en función de la dimensión académica. 


\section{Articulación a lo interno y externo de las unidades académicas}

Diferentes áreas del conocimiento vinculadas a la temática que se va investigar

Direcciones académicas

Facultades; Escuelas, Institutos de Investigación

CUR'S, Institutos Tecnológicos

Departamentos, Carreras
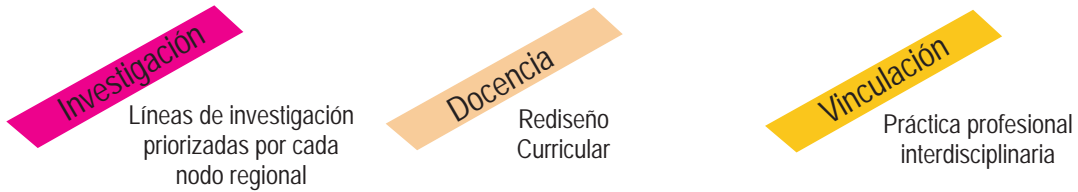

Gráfico 2

Articularse desde la perspectiva del quehacer universitario a lo externo, con las diferentes iniciativas y políticas de desarrollo nacional e internacional; alineamiento de los temas prioritarios regionales con los objetivos de desarrollo del milenio, la estrategia para la reducción de la pobreza y la estrategia de competitividad sistémica en el marco de la Visión de País 2010-2038 y Plan de Nación 2010-2022.

Diseñar e implementar la estrategia de movilización de recursos, en función de las necesidades considerando los umbrales de desempeño optimo.

Implementación efectiva de acciones, para lograr los retos pendientes según las etapas y fases de implementación del ciclo de proyecto de Redes Educativas para impulsar la política de Gestión del Conocimiento en Redes Educativas.

\section{CONCLUSIONES}

Se reducen las limitaciones a medida que se logran los esquemas de gestión compartida, complementariedad y sinérgica. Además los alcances dependerán en gran medida de las articulaciones internas y externas de las unidades académicas, lo que permite generar procesos de aprendizaje por aproximaciones en ciclos de acuerdo a las capacidades propias de las unidades.

La promoción de cambios en los instrumentos regulatorios son procesos que 
requieren de apoyos a todos los niveles y en doble vía. Los avances son lentos si no se logra desarrollar la motivación y satisfacción de los involucrados. Se requiere generar la capacidad instalada en procesos de gestión de todas las áreas del conocimiento con sus particularidades. Urge el establecimiento de mecanismos y estructuras de coordinación y articulación. Así como identificar los temas y áreas prioritarias pertinentes por cada nodo regional.

Para lograr viabilidad económico /administrativa, se requiere una consistencia entre los resultados e impactos que se esperan en el corto, mediano y largo plazo de la política de "Redes Educativas...." y la asignación de recursos (humanos, materiales, logísticos, presupuestario). De acuerdo a lo anterior se pueden considerar varios escenarios de resultados e impactos: Umbral Pobre Desempeño, Umbral Aceptable Desempeño, Umbral Buen Desempeño, Umbral Excelente Desempeño.

Se ha tenido un acompañamiento aceptable, tomando en cuenta los recursos que posee. Aunque falta mucho por recorrer, los fundamentos teóricos de las redes ya están establecidos, lo que permitirá en el futuro inmediato consolidar ese concepto y su gestión. Algunas unidades académicas sostienen que hay desconocimiento del enfoque de redes educativas regionales, primordialmente por falta de socialización y de canales de comunicación inter-unidad académica.

La regionalización de las redes permitirán, por un lado la integración, concreción y potenciación de las líneas de acción de la reforma; por otro lado, una complementariedad de los recursos y potencialidades de las diferentes Unidades académicas 0 estructuras regionales universitarias, considerando los criterios demográficos (densidad poblacional), capacidad económica, estructura universitaria, cuencas, cercanía cultural y geográfica (departamentalización), y disponibilidad logística actual o potencial para organizar las redes. Se pretende con ese propósito la siguiente regionalización de las redes: Región I o Central, Región II u Oriental, Región III o del Caribe, Región IV o del Norte, Región V u Occidental, Región VI o Sur, Región VII o Sur Oriental, Región VIII y Región IX. Por las características particulares, se define como Zona Especial. Ver mapa 2.

\section{BIBLIOGRAFÍA}

COHEP. Centro de Investigaciones Económicas y Sociales. Propuesto de ordenamiento territorial. Tegucigalpa: 2006.

Calderón, R. y Arias. C. Redes Educativas Regionales de la UNAH, para la Gestión 
del Conocimiento, con Calidad, Pertinencia y Equidad. Serie de Publicaciones de la Reforma Universitaria No.2. Tegucigalpa: 2008.

Collison, C. y Parcell, G. Aprendiendo a volar: Gestión del conocimiento práctico de las organizaciones líderes y de enseñanza. Reino Unido: Capstone; 2004.

Egger, U.K. Redes del conocimiento: ¿Qué hace que las redes funcionen? Noticias de las Cuencas. 2003; (25):28-30.

Farago, J. y Skyrme, D.J. Aprendizaje organizacional. 1995. 3ed. [Monografía en internet] New Bury, Reino Unido. Disponible en

\section{www.skyrme.com/insights/3lrnorg.htm\#culture}

Gorman, M.E. Tipos de conocimiento y sus roles en la transferencia de la tecnología. Diario de la transferencia de la tecnología. 2002; 27 (3): 219-231.

Keuls, C. La estructura para la Gestión del conocimiento: La cadena del valor del conocimiento. 6ed. Países Bajos: 2003.

Lorenzoni, G. y Baden-Fuller, C. Creación de un centro estratégico para gestionar una red de asociados. Revisión de la gestión en California. 1995; 37 (3).

Nonaka, I. y Takeuchi, H. Cómo las empresas japonesas crean la dinámica de la innovación. Nueva York: Universidad de Oxford; 1995.

Pels, J. y Odhiambo F. Diseño y experiencias prácticas del módulo de gestión de conocimiento de Learn@WELL. Diario de la Gestión del conocimiento para el desarrollo. 2005; 1(2): 4-18.

Polanyi, M. La dimensión tácita. Londres, Routledge \& Kegan. 1966.

Röling et al. Basisboek voorlichtingskunde. Amsterdam: Boom; 1994.

Senge, P.M. La quinta disciplina: El arte y la práctica del aprendizaje organizacional. Londres: Random House; 1990.

Skyrme, D.J. Cómo desarrollar una estrategia exitosa de la gestión del conocimiento. Newbury, Reino Unido: David Skyrme yAsociados; 2002.

Snowden, D.J. Gestión justo a tiempo. Parte 1. Reconocimiento de errores comunes de la GC y el progreso que dichos errores inhiben. Revisión de la Gestión del Conocimiento. 2002; 5 (5):14-17.

Snowden, D. J. El conocimiento que usted necesita, justo cuando lo necesita: Revisión de la GC. 2003; 5(6).

Stewart, T.A. Capital intelectual: El nuevo patrimonio de las organizaciones. Londres: Brealy; 1997.

Sveiby, K.E. ¿Qué es la gestión del conocimiento? 2001. Disponible en: www.sveiby.com/articles/KnowledgeManagement.html

UNAH. Equipo Técnico del Seminario Permanente de la Reforma Universitaria. Gestión del conocimiento y ejes curriculares en la Escuela de Ciencias de la Salud en la UNAH-VS. San Pedro Sula: 2007

UNAH: Memoria de la Jornada de seguimiento del Centro Universitario Regional de Occidente- CUROC. Santa Rosa de Copán: Universitaria; 2007. 
UNAH. Relatoría del taller sobre Redes Educativas Regionales para la Gestión del Conocimiento con Calidad, Pertinencia y Equidad. Tegucigalpa: 2007.

Visscher, J.T. editor. Transferencia de la tecnología en el sector de suministro de agua y saneamiento: una experiencia de aprendizaje de Colombia. Delft, Países Bajos: Centro Internacional de Agua y Saneamiento IRC; 1997.

Visccher, JT, et al: Knowledge and Information Management in the Water and Sanitation. Delft, Países Bajos: IRC; 2006.

Weggeman, M. Kennismanagement: de praktijk. Schiedam. Holanda: Scriptum; 2000.

Wheatley, M. El verdadero trabajo de la Gestión del conocimiento. Diario IHRIM. 2001; $5(2): 29-33$.

Wilson, T.D. Los desatinos de la 'Gestión del conocimiento. Investigación de la Información. 2002; 8 (1) 144.

\section{ANEXOS}

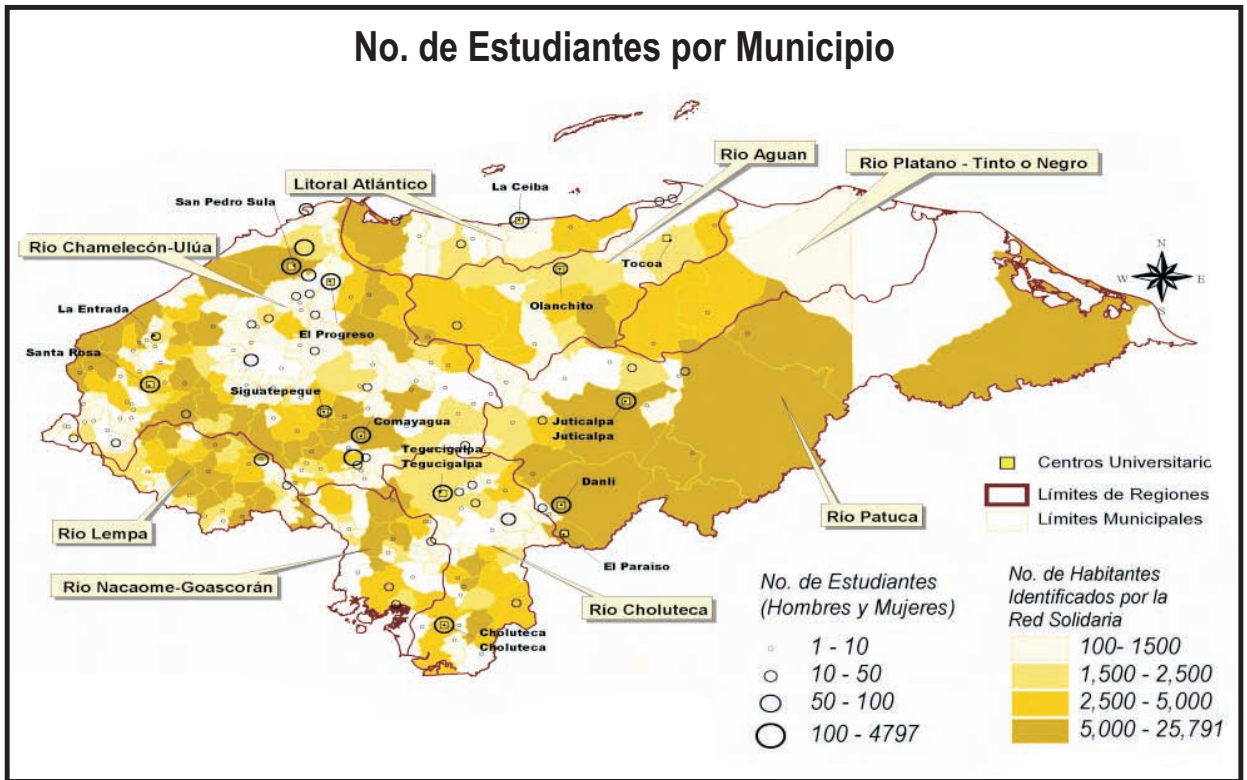

Mapa 1 
DESARROLLO DE LAS REDES

\begin{tabular}{|c|c|}
\hline Etapa I: Diseño e instrumentación & Ejecutado \\
\hline Fase 1: Diseño conceptual de la Red & $100 \%$ \\
\hline Fase 2: Definición de la oferta integral de servicios de cada red. & $30 \%$ \\
\hline $\begin{array}{l}\text { Fase 3: Creación legal de las redes y del marco inter e intra - } \\
\text { institucional para la coordinación }\end{array}$ & $80 \%$ \\
\hline $\begin{array}{l}\text { Fase 4: Diseño, formulación y elaboración de la estrategia de } \\
\text { capacitación y generación de capacidad instalada }\end{array}$ & $25 \%$ \\
\hline Etapa II: implementación estratégica & \\
\hline $\begin{array}{l}\text { Fase 1: Focalización y selección de áreas y temas claves de } \\
\text { intervención (según cada red regional) }\end{array}$ & $30 \%$ \\
\hline Fase 2: Registro de actores claves (aliados y sujetos del proceso) & $30 \%$ \\
\hline Fase 3: Diagnostico -Demanda y Oferta -servicios de Educ .Sup & $25 \%$ \\
\hline $\begin{array}{l}\text { Fase 4: Mapeo, Diagnostico Integral de intervenciones actuales y } \\
\text { análisis de riesgos ( sociales, económicos , culturales ,etc ..) }\end{array}$ & $10 \%$ \\
\hline Fase 5: "Alineación" -intervs . de progs y proyectos (inters -exters ) & $40 \%$ \\
\hline Fase 6: Ejecución de acciones en las áreas prioritarias & $25 \%$ \\
\hline Etapa III: Seguimiento, monitoreo, evaluación y ajuste & \\
\hline
\end{tabular}

Matriz 1: Fuente elaboración propia. Nota: el \% calculado es con enfoque cualitativo

Demanda nivel nacional
Red de Agua y Saneamiento de Honduras (sector interdisciplinario) Pilares fundamentales para sostenibilidad:

A) Tecnológicos , B) Ambientales y naturales

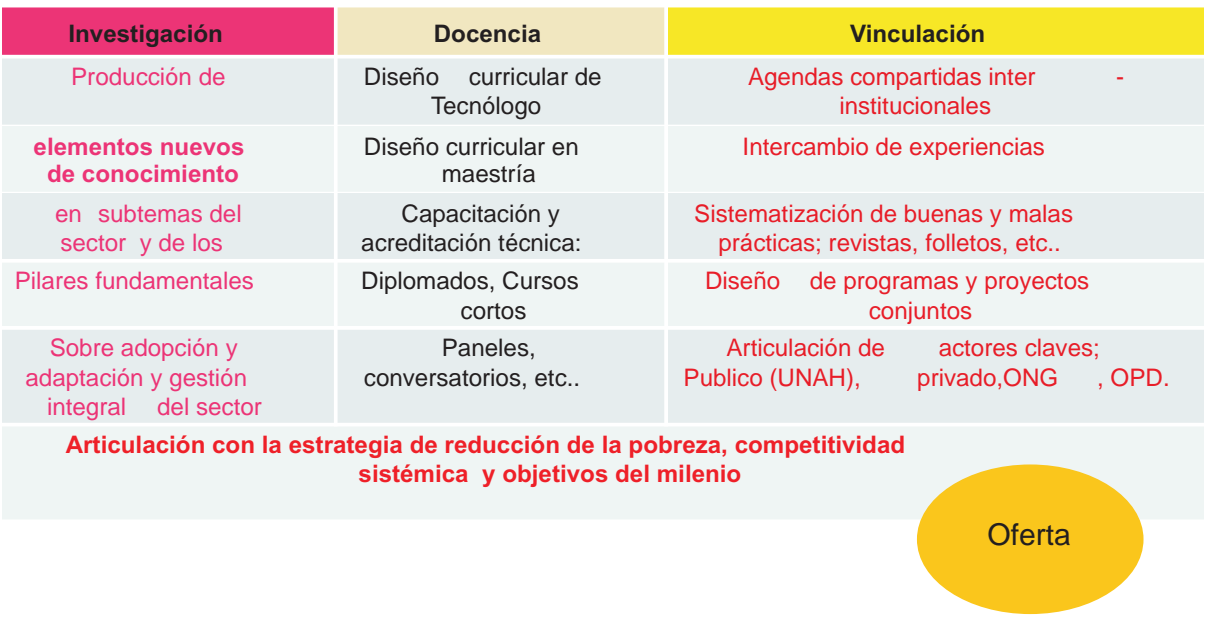

Matriz 2: Fuente Elaboración propia y adaptación basada en los informes de sistematización 